\title{
ROBUST ESTIMATION AND VARIABLE SELECTION FOR PARTIALLY LINEAR ADDITIVE MODEL UNDER MISSING DATA
}

\section{YA-FENG XIA and YU-MEI WANG}

School of Science

Lanzhou University of Technology

Lanzhou 730050

P. R. China

e-mail: gsxyf01@163.com

1421745664@qq.com

\begin{abstract}
In this paper, we investigate the robust estimation and variable selection for partially linear additive model under response missing at random. The nonparametric functions are approximated by B-spline basis. The variable selection of parametric and nonparametric components simultaneously are realized by combining modal regression method and double-SCAD penalty function. We can prove that the variable selection method has Oracle property under some regularity conditions. Some simulation studies are carried out to assess the performance of the proposed methods.
\end{abstract}

2010 Mathematics Subject Classification: 62G08, 62G20.

Keywords and phrases: response missing, modal regression, double-SCAD penalty function, variable selection, robust estimation.

Received September 17, 2018

(ㄷ) 2018 Scientific Advances Publishers 


\section{Introduction}

The general form of partially linear additive model as follows:

$$
Y=X^{T} \beta+\sum_{l=1}^{d} g_{l}\left(Z_{l}\right)+\varepsilon,
$$

where $Y$ is the response variable, $\quad X=\left(x_{1}, \cdots, x_{p}\right)^{T}$ and $Z=\left(z_{1}, \cdots, z_{d}\right)^{T}$ are the covariates of $p$ dimension and $d$ dimension, respectively. $\beta=\left(\beta_{1}, \cdots, \beta_{p}\right)^{T}$ and $g_{l}(\cdot), \cdots, g_{d}(\cdot)$ are $p$ dimensional unknown parameter vectors and $d$ one-dimensional unknown smooth functions, respectively. $\varepsilon$ is a random error and satisfies the condition $E(\varepsilon \mid X, Z)=0$. In the model (1), there is a linear relationship between response the variable $Y$ and the covariate $X$. The relationship between the response variable $Y$ and the covariant variable $Z$ is expressed by the finite degree accumulation after the one dimensional unknown smooth function $g_{l}(\cdot)$ acts on the covariant $Z$.

Model (1) is a typical semi-parametric regression model which combines additive model with linear model. Estimation and variable selection of this kind of model have been studied extensively. In recent years, the problem of semi-parametric model variable selection has become a research topic based on robust regression, Lian et al. [1] considered the high-dimensional variable selection problem of the linear part of the partially linear additive model based on adaptive LASSO penalty, which proved its large sample properties. Based on quantile regression, Noh et al. [2] studied variable selection of varying-coefficient model, and Guo et al. [3] researched the robust variable selection of partially linear additive model by using adaptive LASSO penalty. The mode is a robust statistic because it has a good ability to avoid outliers in the data and can focus on the value of most data. In recent years, the study of robust estimation and variable selection based on the frame of 
mode regression has attracted widely attention from scholars, such as Zhang et al. [4] studied robust estimation and variable selection for partially linear variable coefficient models under the framework of mode regression. Yang [5] studied the robust estimation and variable selection of single index vary-coefficient model under the framework of mode regression. Lv et al. [6] researched robust estimation and variable selection of partially linear additive model based on mode regression.

Variable selection problem for missing data, Zhao [7] has surveied the variable selection problem of partial varying-coefficient model based on the method of imputation-based under the random missing response variable; Yang et al. [8] applied SCAD penalty and adaptive LASSO penalty to study variable selection problem of partially variable coefficient EV model under missing of response variables. Based on quantile regression, Ding et al. [9] studied parameter estimation with missing data model. In this paper, based on [7], the partial linear additive model (1) is considered to study the problem of robust variable selection in the case of random miss of response variables under the mode regression framework. The double SCAD penalty estimation equation is constructed by mode regression, and the variable selection of parameters and non-parametric functions is realized simultaneously. The Oracle property of penalty estimation is proved.

\section{Variable Selection}

Suppose that $\left\{Y_{i}, X_{i}, Z_{i}\right\}_{i}^{n}, i=1,2, \cdots, n$ are independent and identically distributed sample from model (1), where the response variable $Y$ is missing at random and the covariates $X_{i}$ and $Z_{i}$ can be observed completely. If the response variable $Y$ is missing, then make $\delta_{i}=0$, otherwise $\delta_{i}=1$, in other words $P\left(\delta_{i}=1 \mid Y_{i}, X_{i}, Z_{i}\right)=p\left(\delta_{i}=\right.$

$\left.1 \mid X_{i}, Z_{i}\right)$. The model with missing data (1) as follows:

$$
\delta_{i} Y_{i}=\delta_{i} X_{i}^{T} \beta+\delta_{i} \sum_{l=1}^{d} g_{l}\left(Z_{i l}\right)+\delta_{i} \varepsilon_{i} .
$$


Next, we study the problem of variable selection in the parameter and nonparametric part of the model (2) by combining the thought of mode regression of Yao et al. [10] and the idea of imputation-based penalized estimation of Zhao et al. [7]. We first use the B-spline to approximate the non-parametric function $g_{l}(\cdot)$. Suppose $\left\{\psi_{l}(z), \cdots, \psi_{\widetilde{K}_{n}}(z)\right\}$ is a B-spline basis function with $m+1$ order, where $K_{n}=K+m+1$ is the number of base functions and $K$ is the number of inner knot of the base function. At this point, each non-parametric function $g_{l}\left(Z_{l}\right)$ can be approximated by the following basis functions:

$$
g_{l}\left(Z_{l}\right) \approx \sum_{k=1}^{K} \gamma_{l k} \psi_{l k}\left(z_{l}\right)=\psi_{l}^{T}\left(z_{l}\right) \gamma_{l}
$$

where $\psi_{l}\left(z_{l}\right)=\left(\psi_{l 1}\left(z_{l}\right), \cdots, \psi_{l K_{n}}\left(z_{l}\right)\right)^{T}$ and $\gamma_{l}=\left(\gamma_{l 1}, \cdots, \gamma_{l K_{n}}\right)^{T}$ are both B-spline coefficients vectors. Mark $\psi_{i l}=\psi_{l}\left(Z_{i l}\right), l=1, \cdots, d$ and $\Psi_{i}=\left(\psi_{i 1}^{T}, \cdots, \psi_{i d}^{T}\right)^{T}$. In this case, the model (2) is approximately expressed as

$$
\delta_{i} Y_{i}=\delta_{i} X_{i}^{T} \beta+\delta_{i} \Psi_{i} \gamma+\delta_{i} \varepsilon_{i}, \quad i=1, \cdots, n,
$$

where $\gamma=\left(\gamma_{1}^{T}, \cdots, \gamma_{d}^{T}\right)$. Under the mode regression, the spline mode estimation of $\beta$ and $\gamma$ is obtained by the following objective function:

$$
Q(\beta, \gamma)=\phi_{h}\left[\delta_{i}\left(Y_{i}-X_{i}^{T} \beta-\Psi_{i} \gamma\right)\right], \quad i=1, \cdots, n,
$$

where $\Phi_{h}(t)=h^{-1} \Phi(t / h), \Phi_{h}(t)$ is kernel density function, and $h$ is bandwidth. Furthermore, we consider the penalty objective function of the model (3) in the case of random missing response variables by combining the idea of double SCAD penalty and imputation-based penalized, as follows:

$$
\eta(\beta, \gamma)=\sum_{i=1}^{n} \widetilde{Q}(\beta, \gamma)-n \sum_{k=1}^{p} P_{\lambda_{1 k}}\left(\left|\beta_{k}\right|\right)-n \sum_{l=1}^{d} P_{\lambda_{2 l}}\left(\left\|\gamma_{l}\right\|_{H_{l}}\right)
$$


where $\widetilde{Q}(\beta, \gamma)=Q(\beta, \gamma)+\left(1-\delta_{i}\right) m_{Q}\left(Z_{i}, \beta, \gamma\right)$, and $m_{Q}\left(Z_{i}, \beta, \gamma\right)$ is the kernel estimator of $E\left\{Q(\beta, \gamma) \mid Z_{i}=z\right\}$, which is defined as

$$
m_{Q}\left(Z_{i}, \beta, \gamma\right)=\sum_{i=1}^{n} \omega_{n i}(z) Q(\beta, \gamma),
$$

where $\omega_{n i}=K_{h_{1}}\left(z-Z_{i}\right) / \sum_{j=1}^{n} \delta_{j} K_{h_{1}}\left(z-Z_{j}\right), K_{h_{1}}(\cdot)=h_{1}^{-1} K\left(\cdot / h_{1}\right), K(\cdot)$ are kernel function, and $h_{1}>0$ is a bandwidth. $P_{\lambda}(\cdot)$ are SCAD penalty parameters, and $P_{\lambda}(0)=0 . \lambda_{1}$ and $\lambda_{2}$ are the adjustment parameters that control the sparsity of the part of the parameter and the part of the non-parametric function respectively, where $\left\|\gamma_{l}\right\|_{H_{l}}=\left(\gamma_{l}^{T} H_{l} \gamma_{l}\right)^{1 / 2}$ and $H_{l}$ is a $K \times K$ matrix with the $\left(k, k^{\prime}\right)$ entry being $\int_{0}^{1} \psi_{l k}(z) \psi_{l k^{\prime}}(z) d z$. We denote

$$
\begin{aligned}
& a_{n}=\max _{k, l}\left\{\left|p_{\lambda_{1 k}}^{\prime}\left(\left|\beta_{0 k}\right|\right)\right|,\left|p_{\lambda_{2 l}}^{\prime}\left(\left\|\gamma_{0 l}\right\|_{H_{l}}\right)\right|, \beta_{0 k} \neq 0, \gamma_{0 l} \neq 0\right\}, \\
& b_{n}=\max _{k, l}\left\{\left|p_{\lambda_{1 k}}^{\prime \prime}\left(\left|\beta_{0 k}\right|\right)\right|,\left|p_{\lambda_{2 l}}^{\prime \prime}\left(\left\|\gamma_{0 l}\right\|_{H_{l}}\right)\right|, \beta_{0 k} \neq 0, \gamma_{0 l} \neq 0\right\},
\end{aligned}
$$

where $\gamma_{0 l}$ is the best approximation of B-spline coefficient of $g_{l}\left(z_{l}\right)$.

\section{Theoretical Property}

In order to establish asymptotic properties of the proposed estimators, we need the following regular conditions. The $H_{r}$ is the whole of all functions $h(t)$ that meet the following conditions on $[0,1]$, where the $M$-order derivative of $h(l)$ is continuous and it satisfies the $H \ddot{l} l d e r$ condition of the $V$-order.

(C-1) $E\left(g_{l}\left(Z_{l}\right)\right)=0$ and $g_{l}\left(z_{l}\right) \in H_{r}, l=1, \cdots, d, r>1 / 2$. 
(C-2) $Z_{l}$ has a continuous density function $f_{z_{l}}\left(z_{l}\right)$ and there are constants $c_{1}$ and $c_{2}$ so that $f_{z_{l}}\left(z_{l}\right)$ meet the condition $0<c_{1} \leq f_{z_{l}}\left(z_{l}\right) \leq$ $c_{2}<\infty, l=1, \cdots, d$ on the interval $[0,1]$.

(C-3) Within $1 \leq i \leq n$, arbitrary random variable $X_{i}$ is uniformly bounded and the eigenvalues of $E\left\{X_{i} X_{i}^{T} \mid Z_{i}\right\}$ are bounded.

(C-4) $t_{1}, \cdots, t_{k_{n}}$ are internal knots on $[0,1], t_{0}=0, t_{k+1}=1, \xi_{i}=t_{i}$ $-t_{i-1}$ and $\xi=\max \left\{\xi_{i}\right\}$, there is a constant $C_{0}$ that makes $\frac{\xi}{\min \left\{\xi_{i}\right\}} \leq C_{0}$, $\max \left\{\left|\xi_{i+1}-\xi_{i}\right|\right\}=o\left(k_{n}^{-1}\right)$.

(C-5) $F(x, z, h)$ and $G(x, z, h)$ continuous on $(x, z)$, and $F(x, z, h)<0$, for $\forall h>0$.

(C-6) The random error $\varepsilon$ satisfies $E\left(\phi_{h}^{\prime}(\delta \varepsilon) \mid X=x, Z=z\right)=0, E$ $\left(\phi_{h}^{\prime \prime}(\delta \varepsilon)^{2} \mid X=x, Z=z\right), E\left(\phi_{h}^{\prime}(\delta \varepsilon)^{3} \mid X=x, Z=z\right) \quad$ and $\quad E\left(\phi_{h}^{\prime \prime \prime}(\delta \varepsilon) \mid X=x\right.$, $Z=z$ ), and it is continuous with respect to the variable $x$ and $z$.

(C-7) $\liminf \operatorname{infm}_{n \rightarrow \infty} \liminf _{\beta_{k} \rightarrow 0^{+}} \lambda_{1 k}^{-1} P_{\lambda_{1 k}}^{\prime}\left(\beta_{k}\right)>0, k=s+1, \cdots, p$ and

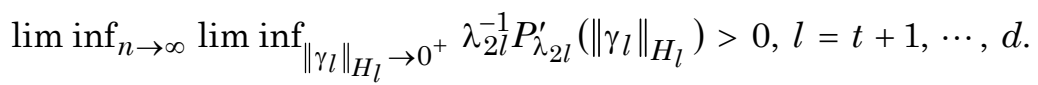

(C-8) $b_{n} \rightarrow 0$, if $n \rightarrow \infty$.

(C-9) Let $n \rightarrow \infty$. The kernel estimates $m_{Q}(z, \gamma, \beta)$ bandwidth of $h_{1}$ satisfying $n h_{1}^{2} \rightarrow \infty$, and $n h_{1}^{4} \rightarrow 0$.

The asymptotic properties of penalty estimators $\hat{\gamma}$ and $\hat{\beta}$ will be given under regular conditions. We define $\beta_{0}$ and $g_{0 l}\left(z_{l}\right)$ as the truth values of $\beta, g_{l}\left(z_{l}\right)$, respectively. Without loss of generality, we premise $\beta_{0 k} \neq 0, k=1, \cdots, s$ and $\beta_{0 k}=0, k=s+1, \cdots, p$, and premise $g_{0 l} \neq 0$, 
$l=1, \cdots, t, g_{0 l}=0, l=t+1, \cdots, d$. Let $\beta=\left(\beta_{a}^{T}, \beta_{b}^{T}\right), \gamma=\left(\gamma_{a}^{T}, \gamma_{b}^{T}\right)$, where $\beta_{a}=\left(\beta_{1}^{T}, \cdots, \beta_{s}^{T}\right)^{T}, \gamma_{a}=\left(\gamma_{1}^{T}, \cdots, \gamma_{t}^{T}\right)^{T}$. Therefore, the corresponding covariates become $X_{a}$ and $\Psi_{a}, \beta_{0 a}$ be the true values of $\beta$. We define

$$
\begin{gathered}
F(x, z, h)=E\left\{\phi_{h}^{\prime \prime}(\delta \varepsilon) \mid X=x, Z=z\right\}, G(x, z, h)=E\left\{\phi_{h}^{\prime}(\delta \varepsilon)^{2} \mid X=x, Z=z\right\}, \\
\Phi=E\left(\phi_{h}^{\prime \prime}(\delta \varepsilon) \Psi \Psi^{T}\right)=E\left\{F(X, Z, h) \Psi \Psi^{T}\right\} . \\
\Xi=E\left(\phi_{h}^{\prime \prime}(\delta \varepsilon) \Psi X^{T}\right)=E\left\{F(X, Z, h) \Psi X^{T}\right\} .
\end{gathered}
$$

Theorem 3.1. Suppose that the regularity conditions (C-1)-(C-9) were established. The numbers of knot $K$ satisfies $K=O\left(n^{1 /(2 r+1)}\right)$, we have

(i) $\left\|\hat{\beta}-\beta_{0}\right\|=O_{p}\left(n^{\frac{-r}{2 r+1}}+a_{n}\right)$,

(ii) $\left\|\hat{g}_{l}-g_{0 l}\right\|=O_{p}\left(n^{\frac{-r}{2 r+1}}+a_{n}\right), l=1, \cdots, d$.

Theorem 3.1 gives the consistency of $\hat{\gamma}$ and $\hat{g}_{l}\left(z_{l}\right)$. In the following, we give the sparsity of penalty estimation and the asymptotic properties of non-zero parameter estimation.

Theorem 3.2. Suppose that the regularity conditions (C-1)-(C-9) were established. The numbers of knot $K$ satisfies $K=O\left(n^{1 /(2 r+1)}\right)$, let $\lambda_{\max }$ $=\max _{k, l}\left\{\lambda_{1 k}, \lambda_{2 l}\right\}$ and $\lambda_{\min }=\min _{k, l}\left\{\lambda_{1 k}, \lambda_{2 l}\right\}$, if $n \rightarrow \infty, \lambda_{\max } \rightarrow 0$ and $n^{r /(2 r+1)} \lambda_{\text {min }} \rightarrow \infty$ has the penalty estimate $\hat{\beta}$ and $\hat{g}_{l}$ with probability 1 satisfies

(i) $\hat{\beta}_{k}=0, k=s+1, \cdots, p$,

(ii) $\hat{g}_{l}=0, l=t+1, \cdots, d$. 
Theorem 3.3. Under the conditions of Theorem 3.2, the linear portion having a non-zero parameter

$$
\sqrt{n}\left(\hat{\beta}_{a}-\beta_{0 a}\right) \rightarrow N\left(0, \sum_{a}^{-1} \Omega_{a} \sum_{a}^{-1}\right)
$$

where $\sum_{a}$ and $\Omega_{a}$ are the first $t \times t$ sub-matrices of $\sum$ and $\Omega$, respectively. $\quad \sum=\left\{F(X, Z, h) \check{X} \check{X}^{T}\right\}, \Omega=\left\{G(X, Z, h) \check{X} \check{X}^{T}\right\}, \check{X}=X$ $-\Xi^{T} \Phi^{-1} \Psi$.

\section{Algorithm and Selection of Regularization Parameters}

\subsection{Algorithm}

It is difficult to directly maximize the penalty objective function. Referring to the local quadratic approximation (LQA) method in Fan [11] and the EM algorithm, this paper gives the implementation steps of the penalty estimation algorithm. Assumed that initial estimates $\beta_{k}^{(0)}$ and $\gamma_{l}^{(0)}$, if $\left|\beta_{k}^{(0)}\right|>0$ and $\left\|\gamma_{l}^{(0)}\right\|_{H_{l}}>0$. Based on LQA thought, we can get

$$
\begin{gathered}
p_{\lambda_{1 k}}\left(\left|\beta_{k}\right|\right) \approx p_{\lambda_{1 k}}\left(\left|\beta_{k}^{(0)}\right|\right)+\frac{1}{2} \frac{p_{\lambda_{1 k}}^{\prime}\left(\left|\beta_{k}^{(0)}\right|\right)}{\left|\beta_{k}^{(0)}\right|}\left(\left|\beta_{k}\right|^{2}-\left|\beta_{k}^{(0)}\right|^{2}\right), \\
p_{\lambda_{2 l}}\left(\left\|\gamma_{l}\right\|_{H_{l}}\right) \approx p_{\lambda_{2 l}}\left(\left\|\gamma_{l}^{(0)}\right\|_{H_{l}}\right)+\frac{1}{2} \frac{p_{\lambda_{2 l}}^{\prime}\left(\left\|\gamma_{l}^{(0)}\right\|_{H_{l}}\right)}{\left\|\gamma_{l}^{(0)}\right\|_{H_{l}}}\left(\left\|\gamma_{l}\right\|_{H_{l}}^{2}-\left\|\gamma_{l}^{(0)}\right\|_{H_{l}}^{2}\right) .
\end{gathered}
$$

We denote $\theta=\left(\beta^{T}, \gamma^{T}\right)^{T}, S_{i}=\left(X_{i}^{T}, \Psi_{i}^{T}\right), m=0$,

$$
\begin{aligned}
& \sum_{\lambda_{1}, \lambda_{2}}\left(\theta^{(m)}\right)=\operatorname{diag} \\
& \left\{\frac{p_{\lambda_{11}}^{\prime}\left(\left|\beta_{1}^{(m)}\right|\right)}{\left|\beta_{1}^{(m)}\right|}, \cdots, \frac{p_{\lambda_{1 p}}^{\prime}\left(\left|\beta_{p}^{(m)}\right|\right)}{\left|\beta_{p}^{(m)}\right|}, \frac{p_{\lambda_{21}}^{\prime}\left(\left\|\gamma_{1}^{(m)}\right\|_{H_{1}}\right)}{\left\|\gamma_{1}^{(m)}\right\|_{H_{1}}} H_{1}, \cdots, \frac{p_{\lambda_{2 d}}^{\prime}\left(\left\|\gamma_{d}^{(m)}\right\|_{H_{d}}\right)}{\left\|\gamma_{d}^{(m)}\right\|_{H_{d}}} H_{d}\right\} .
\end{aligned}
$$


The following is a penalty estimate for the penalty objective function (6) under the random missing response variable $Y$ based on the EM algorithm.

Step 1 (E-step): Update $\pi\left(i \mid \theta^{(m)}\right)$ by

$$
\begin{gathered}
\pi\left(i \mid \theta^{(m)}\right)=\frac{\widetilde{Q}_{i}(\beta, \gamma)}{\sum_{i=1}^{n} \widetilde{Q}_{i}(\beta, \gamma)} \\
=\frac{\phi_{h}\left[\delta_{i}\left(Y_{i}-S_{i} \theta^{(m)}\right)\right]+\left(1-\delta_{i}\right) \sum_{i=1}^{n} \omega_{n i}(u) \phi_{h}\left[\delta_{i}\left(Y_{i}-S_{i} \theta^{(m)}\right)\right]}{\sum_{i=1}^{n}\left\{\phi_{h}\left[\delta_{i}\left(Y_{i}-S_{i} \theta^{(m)}\right)\right]+\left(1-\delta_{i}\right) \sum_{i=1}^{n} \omega_{n i}(u) \phi_{h}\left[\delta_{i}\left(Y_{i}-S_{i} \theta^{(m)}\right)\right]\right\}} .
\end{gathered}
$$

Step 2 (M-step): Update $\hat{\theta}^{(m+1)}$ by

$$
\begin{aligned}
\hat{\theta}^{(m+1)} & =\arg \max _{\theta} \sum_{i=1}^{n}\left\{\pi\left(i \mid \theta^{(m)}\right) \log \widetilde{Q}_{i}(\gamma, \beta)\right\}+\frac{n}{2} \theta^{T} \sum_{\lambda_{1}, \lambda_{2}}\left(\theta^{(m)}\right) \theta \\
& \approx\left(\delta^{2} S^{T} W S+n \sum_{\lambda_{1}, \lambda_{2}}\left(\theta^{(m)}\right)\right)^{-1} \delta^{2} S^{W} Y
\end{aligned}
$$

where $S=\left(S_{1}, \cdots, S_{n}\right), W$ is an $n \times n$ diagonal matrix with diagonal elements $\pi\left(i \mid \theta^{(m)}\right)$.

Step 3: Repeat E-step and M-step iteratively until the algorithm converges. The $\hat{\theta}$ be the estimated value of $\theta$, then there are $\hat{\beta}=\left(I_{p \times p}\right.$, $\left.0_{p \times p \times q K_{n}}\right) \hat{\theta}, \hat{\gamma}=\left(0_{d K_{n} \times p}, I_{d K_{n} \times d K_{n}}\right) \hat{\theta}, \hat{g}_{l}=\psi_{l}\left(z_{l}\right)^{T} \hat{\gamma}_{l}, l=1, \cdots, d$.

\subsection{Selection of regularization parameters}

In realistic simulations, it is often necessary to select appropriate adjustment parameters to obtain a better sparse estimate. In this paper, cubic spline $(m=3)$ is used to approximate the part of non-parametric function, the number of internal knot is $K=2 m+3$ and it is equidistant 
node. Learn from Zhao et al. [12] approached and used the following way to select tuning parameters

$$
\lambda_{1 k}=\frac{\lambda_{1}}{\left|\widetilde{\beta}_{k}\right|}, \lambda_{2 l}=\frac{\lambda_{2}}{\left\|\widetilde{\gamma}_{l}\right\|_{H_{l}}}
$$

where $\widetilde{\beta}_{k}$ and $\widetilde{\gamma}_{l}$ are non-penalty estimate of $\beta_{k}$ and $\gamma_{l}$. The adjustment parameters $\lambda_{1}$ and $\lambda_{2}$ are selected through the BIC criterion.

$$
\begin{aligned}
\operatorname{BIC}\left(\lambda_{1}, \lambda_{2}\right)=-\frac{1}{n} \sum_{i=1}^{n} \Phi_{h}\left\{\delta_{i}\left[Y_{i}-X_{i}^{T} \hat{\beta}_{\lambda_{1}}-\Psi_{i}^{T} \hat{\gamma}_{\lambda_{2}}\right]\right\} & +d f_{n} \frac{\log (n / K)}{n / K} \\
& +d f_{c} \frac{\log (n)}{n},
\end{aligned}
$$

where $\hat{\beta}_{\lambda_{1}}$ and $\hat{\gamma}_{\lambda_{2}}$ represents a penalty at a given estimate $\lambda_{1}$ and $\lambda_{2}, d f_{n}$ is the number of non-zero additive function, and $d f_{c}$ is the number of non-zero parameters.

\section{Simulation Studies}

In the model (1), we assume that $\beta=(2.5,1.5,0.5,1.8,0,0,0,0,0,0)^{T}$, $g_{l}(z)=\left(g_{1}(z), \cdots, g_{10}(z)\right)^{T}, g_{1}(z)=5.5+0.1 \exp (2 z-1), g_{2}(z)=2.5-\sin (\pi z)$, $g_{3}(z)=1.5+z(1-z)$, and the regression coefficients of other unrelated variables in the model are taken as 0 . To implement the simulation, we take covariates $X_{i} \sim N(0,1), i=1, \cdots, 10, Z \sim U(0,1)$, and $Y$ is generated by the model, where $\varepsilon \sim N(0,0.5)$. In this paper, we choose the probability function $\Delta(z)=P(\delta=1 \mid Z=z)$ to be $\Delta_{1}(z) \equiv 0.5$, $\Delta_{2}(z)=\frac{e^{z}}{0.7+e^{z}}$, and $\Delta_{3}(z)=0.9+0.2(z-0.5)$, respectively. In about three cases, the corresponding probability of deletion is about $0.5,0.3$, and 0.1 , respectively. In the simulation, we take sample capacity $n=200$, $n=400, n=600$ to simulate repeatedly. In addition, the third-order 
B-spline basis functions are used to approximate the variable coefficient functions, and the number of the internal knot $K$ and the adjustment parameters $\lambda_{1}, \lambda_{2}$ are selected through the BIC criterion method. Three different methods of variable selection are compared and analyzed under the regression of mode: (1) based on imputed estimating equations in this paper (MISCAD); (2) based on the full data set (MFSCAD); and (3) based on ignoring the missing data set (MCSCAD). For visual comparison, we use the square root of average square error (RASE) to evaluate the effect of non-parametric function estimation, which is defined

$$
R A S E=\left\{\frac{1}{N} \sum_{i=1}^{N} \sum_{l=1}^{d}\left[\hat{g}_{l}\left(z_{i l}\right)-g_{l}\left(z_{i l}\right)\right]^{2}\right\}^{\frac{1}{2}},
$$

and use generalized mean square error (GMSE) to evaluate the estimation accuracy of parameter components estimated by $\hat{\beta}$, which is defined

$$
G M S E=(\hat{\beta}-\beta)^{T} E\left(X X^{T}\right)(\hat{\beta}-\beta) .
$$

During the simulation, "C" indicates the correct estimate of the average number of zero coefficients of 0 , and "I" means the error estimates the average number of non-zero coefficient functions to 0 . The simulation results of the sample size $n=200, n=400, n=600$ parameter component and non-parameter component are given in Table 5.1 and Table 5.2, respectively.

From Table 5.1 and Table 5.2, the following conclusions are drawn: (1) With the increase of sample size $n$, the simulation effect of the three variable selection methods are significantly redounded under the regression of mode. The main reason for this is that the likelihood of outliers in the sample data is greater, but the mode regression can more select the sample value near the truth so that the error is reduced more greatly. (2) With the decrease of missing probability, the error accuracy of parameters and non-parametric parts is gradually reduced, under three 
variable selection methods, and the simulation results of MISCAD method presented in this paper are better than the simulation results of MCSCAD method. Therefore, it illustrates the superiority of the imputation-based method proposed in this paper. (3) Under the model of mode regression, the simulation results based on the MFSCAD method are the best, and the simulation results obtained by the MISCAD method are closer to it, which indicates that the imputation-based penalized estimation variable selection method is effective.

Table 5.1. Simulation results of parameters based on different variable selection methods

\begin{tabular}{cccccccccccc}
\hline & & \multicolumn{3}{c}{$1-E\left(\Delta_{1}(u)\right)=0.5$} & \multicolumn{2}{c}{$1-E\left(\Delta_{2}(u)\right)=0.3$} & \multicolumn{2}{c}{$1-E\left(\Delta_{3}(u)\right)=0.1$} \\
\cline { 3 - 12 }$n$ & & Methods & C & I & GMSE & C & I & GMSE & C & I & GMSE \\
\hline \multirow{2}{*}{200} & MISCAD & 4.8000 & 0.0000 & 0.1135 & 4.8500 & 0.0200 & 0.0946 & 4.9000 & 0.0000 & 0.0760 \\
& MCSCAD & 4.5800 & 0.0000 & 0.1615 & 4.7000 & 0.0400 & 0.1309 & 4.8600 & 0.0000 & 0.1155 \\
& MFSCAD & 4.8200 & 0.0400 & 0.0982 & 4.9000 & 0.0200 & 0.0829 & 4.9400 & 0.0000 & 0.0710 \\
\hline \multirow{2}{*}{400} & MISCAD & 5.8600 & 0.0200 & 0.0368 & 5.9200 & 0.0000 & 0.0293 & 5.9200 & 0.0000 & 0.0192 \\
& MCSCAD & 5.7000 & 0.0000 & 0.0495 & 5.7200 & 0.0000 & 0.0438 & 5.9000 & 0.0000 & 0.0385 \\
& MFSCAD & 5.8200 & 0.0000 & 0.0312 & 5.9000 & 0.0000 & 0.0266 & 5.9800 & 0.0000 & 0.0188 \\
\hline & MISCAD & 5.9400 & 0.0000 & 0.0215 & 6.0000 & 0.0000 & 0.0167 & 5.9800 & 0.0000 & 0.0122 \\
600 & MCSCAD & 5.9200 & 0.0000 & 0.0292 & 5.9400 & 0.0000 & 0.0271 & 5.9800 & 0.000 & 0.0233 \\
& MFSCAD & 5.9600 & 0.0000 & 0.0181 & 5.9800 & 0.0000 & 0.0137 & 6.0000 & 0.0000 & 0.0107 \\
\hline
\end{tabular}


Table 5.2. Simulation results of non-parametric function based on different variable selection methods

\begin{tabular}{cccccccccccc}
\hline & & \multicolumn{3}{c}{$1-E\left(\Delta_{1}(u)\right)=0.5$} & \multicolumn{2}{c}{$1-E\left(\Delta_{2}(u)\right)=0.3$} & \multicolumn{2}{c}{$1-E\left(\Delta_{3}(u)\right)=0.1$} \\
\cline { 3 - 12 }$n$ & & Methods & $\mathrm{C}$ & $\mathrm{I}$ & RASE & $\mathrm{C}$ & $\mathrm{I}$ & RASE & $\mathrm{C}$ & $\mathrm{I}$ & RASE \\
\hline \multirow{2}{*}{200} & MISCAD & 4.2200 & 0.0800 & 0.0576 & 4.2400 & 0.0000 & 0.0529 & 4.600 & 0.0000 & 0.0505 \\
& MCSCAD & 4.0000 & 0.0000 & 0.0591 & 4.3600 & 0.0000 & 0.0581 & 4.5600 & 0.0200 & 0.0545 \\
& MFSCAD & 4.5600 & 0.0000 & 0.0538 & 4.7000 & 0.0000 & 0.0521 & 4.7400 & 0.0000 & 0.0492 \\
\hline \multirow{2}{*}{400} & MISCAD & 6.6000 & 0.0000 & 0.0313 & 6.7600 & 0.0000 & 0.0287 & 6.6400 & 0.0200 & 0.0274 \\
& MCSCAD & 6.3600 & 0.0000 & 0.0317 & 6.4000 & 0.0000 & 0.0308 & 6.6000 & 0.0200 & 0.0300 \\
& MFSCAD & 6.5400 & 0.0000 & 0.0297 & 6.5600 & 0.0200 & 0.0275 & 6.6200 & 0.0000 & 0.0277 \\
\hline & MISCAD & 6.9600 & 0.0400 & 0.0213 & 6.9800 & 0.0400 & 0.0202 & 7.0000 & 0.0000 & 0.0122 \\
600 & MCSCAD & 6.8600 & 0.0200 & 0.0221 & 6.9400 & 0.0200 & 0.0215 & 6.9000 & 0.010 & 0.0233 \\
& & MFSCAD & 6.9800 & 0.0000 & 0.0205 & 7.0000 & 0.0000 & 0.0201 & 7.0000 & 0.0000 & 0.0107 \\
\hline
\end{tabular}

\section{Proof of Theorems}

Proof of Theorem 3.1. Suppose $g_{n l}\left(z_{l}\right)=\psi_{l}^{T} \gamma_{0 l}, l=1, \cdots, d$ is the optimum approximating spline function for $g_{0 l}\left(z_{l}\right)$. According to the result of de Boor [13], if $g_{0 l}\left(z_{l}\right)$ satisfy (C-1), have

$$
\sup _{z_{l} \in[0,1]}\left|g_{n l}\left(z_{l}\right)-g_{0 l}\left(z_{l}\right)\right| \leq C K^{-r}
$$

Let $\tau=n^{-r / 2 r+1}+a_{n}, v_{1}=\tau^{-1}\left(\beta-\beta_{0}\right), v_{2}=\tau^{-1}\left(\gamma-\gamma_{0}\right)$, and $V=\left(v_{1}^{T}, v_{2}^{T}\right)$.

Next, we prove that for any given $\epsilon>0$ store, a sufficiently large positive $C$ can be established.

$$
P\left\{\sup _{\|V\|=C} \eta(\beta, \gamma)<\eta\left(\beta_{0}, \gamma_{0}\right)\right\} \leq 1-\epsilon .
$$


Let $\Delta(\beta, \gamma)=\frac{1}{K}\left\{\eta(\beta, \gamma)-\eta\left(\beta_{0}, \gamma_{0}\right)\right\}$. Using Taylor expansion, there are $\Delta(\beta, \gamma)=\frac{1}{K} \sum_{i=1}^{n}\left\{Q_{i}(\beta, \gamma)-Q_{i}\left(\beta_{0}, \gamma_{0}\right)\right\}+\frac{1}{K} \sum_{i=1}^{n}\left(1-\delta_{i}\right)\left\{m_{Q}\left(Z_{i}, \beta, \gamma\right)-m_{Q}\left(Z_{i}, \beta_{0}, \gamma_{0}\right)\right\}$

$$
\begin{aligned}
& -\frac{n}{K} \sum_{k=1}^{p}\left\{P_{\lambda_{1 k}}\left(\left|\beta_{k}\right|\right)-P_{\lambda_{1 k}}\left(\left|\beta_{0 k}\right|\right)\right\}-\frac{n}{K} \sum_{l=1}^{d}\left\{P_{\lambda_{2 l}}\left(\left\|\gamma_{l}\right\|_{H_{l}}\right)-P_{\lambda_{2 l}}\left(\left\|\gamma_{l 0}\right\|_{H_{l}}\right)\right\} \\
& \triangleq I_{1}+I_{2}-I_{3}-I_{4} .
\end{aligned}
$$

The following is the Taylor expansion of $I_{1}$

$$
\begin{aligned}
I_{1}= & \frac{1}{K} \sum_{i=1}^{n}\left\{\phi_{h}\left[\delta_{i}\left(Y_{i}-X_{i}^{T} \beta-\Psi_{i}^{T} \gamma\right)\right]-\phi_{h}\left[\delta_{i}\left(Y_{i}-X_{i}^{T} \beta_{0}-\Psi_{i}^{T} \gamma_{0}\right)\right]\right\} \\
= & \frac{-\tau}{K} \sum_{i=1}^{n} \delta_{i}\left\{\phi_{h}^{\prime}\left[\delta_{i}\left(\varepsilon_{i}+1_{d}^{T} R\left(Z_{i}\right)\right)\right]\right\}\left(\Psi_{i}^{T} V_{2}+X_{i}^{T} V_{1}\right) \\
& +\frac{\tau^{2}}{K} \sum_{i=1}^{n} \delta_{i}^{2}\left\{\phi_{h}^{\prime \prime}\left[\delta_{i}\left(\varepsilon_{i}+1_{d}^{T} R\left(Z_{i}\right)\right)\right]\right\}\left(\Psi_{i}^{T} V_{2}+X_{i}^{T} V_{1}\right)^{2} \\
& +\frac{\tau^{3}}{K} \sum_{i=1}^{n} \delta_{i}^{3}\left\{\phi_{h}^{\prime \prime \prime}\left(\delta_{i} \epsilon_{i}^{*}\right)\right\}\left(\Psi_{i}^{T} V_{2}+X_{i}^{T} V_{1}\right)^{3} \\
\triangleq & I_{11}+I_{12}+I_{13},
\end{aligned}
$$

where $\delta_{i} \epsilon_{i}^{*}$ is between $\delta_{i}\left[\varepsilon_{i}+1_{d}^{T} R\left(Z_{i}\right)\right]$ and $\delta_{i}\left[\varepsilon_{i}+1_{d}^{T} R\left(Z_{i}\right)-\tau\left(\psi_{i}^{T} V_{2}+\right.\right.$ $\left.\left.X_{i}^{T} V_{1}\right)\right] 1$ is a $d$-dimensional column vector whose elements are all 1 , $R\left(Z_{i}\right)=\left(R_{1}\left(Z_{i 1}\right), \cdots, R_{d}\left(Z_{i d}\right)\right)^{T}, \quad$ and $\quad R_{l}\left(Z_{i l}\right)=g_{0 l}\left(Z_{i l}\right)-\psi_{i l}^{T} \gamma_{0 l}$, $l=1, \cdots, d$. Using the Taylor display for $I_{1}$, we can get

$$
\begin{array}{r}
I_{11}=\frac{-\tau}{K} \sum_{i=1}^{n} \delta_{i}\left\{\phi_{h}^{\prime}\left(\delta_{i} \varepsilon_{i}\right)+\delta_{i} \phi_{h}^{\prime \prime}\left(\delta_{i} \varepsilon_{i}\right) 1_{d}^{T} R\left(Z_{i}\right)+\frac{1}{2} \delta_{i}^{2} \phi_{h}^{\prime \prime \prime}\left(\delta_{i} \zeta_{i}^{*}\right)\left[1_{d}^{T} R\left(Z_{i}\right)\right]^{2}\right\} \\
\times\left(\Psi_{i}^{T} V_{2}+X_{i}^{T} V_{1}\right),
\end{array}
$$


where $\delta_{i} \zeta_{i}^{*}$ is between $\delta_{i} \varepsilon_{i}$ and $\delta_{i}\left[\varepsilon_{i}+1_{d}^{T} R\left(Z_{i}\right)\right]$. By using formula (6), we can get the $\left\|R\left(Z_{i l}\right)\right\|=O\left(K^{-r}\right)$, combined with regular conditions (C-4) and (C-5), can be obtained by simple calculation

$$
I_{11}=O_{p}\left(n K^{-1} \tau K^{-r}\|V\|\right)=O_{p}\left(n \tau^{2} K^{-1}\|V\|\right) .
$$

For $I_{12}, I_{13}$ is equally rational

$$
I_{12}=E(F(X, Z, h)) O_{p}\left(n \tau^{2} K^{-1}\|V\|^{2}\right), I_{13}=O_{p}\left(n \tau^{3} K^{-1}\|V\|^{3}\right) .
$$

Therefore, for sufficiently large positive integer $C$, when $\|V\|=C, I_{12}$ uniformly controls $I_{11}$. According to the penalty property of SCAD, when $\lambda_{\max } \rightarrow 0$, there is $a_{n}=0$. Because $\tau=n^{-r /(2 r+1)} \rightarrow 0$, there is $\tau\|V\| \rightarrow 0$. So we can get $I_{13}=o_{p}\left(I_{12}\right)$, that is, on $\|V\|=C, I_{13}$ is also controlled by $I_{12}$ uniformly. In addition, using a similar method to $I_{1}$, we can deal with $I_{2}$.

$$
\begin{aligned}
I_{2}= & \frac{1}{K} \sum_{i=1}^{n}\left(1-\delta_{i}\right) \omega_{n i}(z)\left\{\phi_{h}\left[\delta_{i}\left(Y_{i}-X_{i}^{T} \beta-\Psi_{i}^{T} \gamma\right)\right]-\phi_{h}\left[\delta_{i}\left(Y_{i}-X_{i}^{T} \beta_{0}-\Psi_{i}^{T} \gamma_{0}\right)\right]\right\} \\
= & \frac{-\tau}{K} \sum_{i=1}^{n}\left(1-\delta_{i}\right) \omega_{n i}(z) \delta_{i}\left\{\phi_{h}^{\prime}\left[\delta_{i}\left(\varepsilon_{i}+1_{d}^{T} R\left(Z_{i}\right)\right)\right]\right\}\left(\Psi_{i}^{T} V_{2}+X_{i}^{T} V_{1}\right) \\
& +\frac{\tau^{2}}{K} \sum_{i=1}^{n}\left(1-\delta_{i}\right) \omega_{n i}(z) \delta_{i}^{2}\left\{\phi_{h}^{\prime \prime}\left[\delta_{i}\left(\varepsilon_{i}+1_{d}^{T} R\left(Z_{i}\right)\right)\right]\right\}\left(\Psi_{i}^{T} V_{2}+X_{i}^{T} V_{1}\right)^{2} \\
& +\frac{\tau^{3}}{K} \sum_{i=1}^{n}\left(1-\delta_{i}\right) \omega_{n i}(z) \delta_{i}^{3}\left\{\phi_{h}^{\prime \prime \prime}\left(\delta_{i} \epsilon_{i}^{*}\right)\right\}\left(\Psi_{i}^{T} V_{2}+X_{i}^{T} V_{1}\right)^{3} \\
\triangleq & I_{21}+I_{23}+I_{23} .
\end{aligned}
$$

We still use the Taylor expansion method to deal with $I_{11}, I_{12}, I_{13}$, and we can get $I_{21}=O_{p}\left(n \tau^{2} K^{-1}\|V\|\right), I_{22}=E(F(X, Z, h)) O_{p}\left(n \tau^{2} K^{-1}\|V\|^{2}\right)$, and $I_{13}=O_{p}\left(n \tau^{3} K^{-1}\|V\|^{3}\right)$. So $I_{21}, I_{23}$ are also dominated by $I_{22}$. 
Based on $p_{\lambda}(0)=0$, we use the Taylor display for $I_{3}$, we have

$$
\begin{aligned}
I_{3} & =\frac{n}{K} \sum_{k=1}^{d}\left\{p_{\lambda_{1 k}}\left(\left|\beta_{k}\right|\right)-p_{\lambda_{1 k}}\left(\left|\beta_{0 k}\right|\right)\right\} \\
& \leq \sum_{k=1}^{s}\left[K^{-1} n \tau p_{\lambda_{1 k}}^{\prime}\left(\left|\beta_{0 k}\right|\right) \operatorname{sgn}\left(\beta_{0 k}\right)\left|v_{1 k}\right|+K^{-1} n \tau^{2} p_{\lambda_{1 k}^{\prime \prime}}\left(\left|\beta_{0 k}\right|\right) \operatorname{sgn}\left(\beta_{0 k}\right)\right. \\
& \leq \sqrt{s} K^{-1} n \tau a_{n}\|v\|+K^{-1} n \tau^{2} b_{n}\|v\|^{2} .
\end{aligned}
$$

According to the conditions $b_{n} \rightarrow 0$, for the full large $C$, when $\|V\|=C, I_{4}$ is consistently controlled by $I_{12}$. Using the same method, it can be proved that $I_{3}$ is also controlled by $I_{12}$. From the regular condition (C-5), there is $F(x, z, h)<0$. By choosing a sufficiently large $C$, the formula (7) holds, and there is a local maximum point of hateta, hatgamma, which makes the following formula hold:

$$
\left\|\hat{\beta}-\beta_{0}\right\|=O_{p}(\tau),\left\|\hat{\gamma}-\gamma_{0}\right\|=O_{p}(\tau)
$$

That is the first part of Theorem 3.1 of the conclusions of the establishment, then the second part of the proof of Theorem 3.1. Noting

$$
\begin{aligned}
\left\|\hat{g}_{l}(\cdot)-g_{0 l}(\cdot)\right\|^{2} & =\int_{0}^{1}\left|\hat{g}_{l}(\cdot)-g_{0 l}(\cdot)\right|^{2} d z \\
& =\int_{0}^{1}\left\{\psi_{l}^{T}(z) \hat{\gamma}_{l}-\psi_{l}^{T}(z) \gamma_{0 l}+R_{l}(z)\right\}^{2} d z \\
& \leq 2 \int_{0}^{1}\left\{\psi_{l}^{T}(u) \hat{\gamma}_{k}-\psi_{l}^{T}(u) \gamma_{k}\right\}^{2} d z+2 \int_{0}^{1} R_{l}(z)^{2} d z \\
& =2\left(\hat{\gamma}_{l}-\gamma_{0 l}\right)^{T} H_{l}\left(\hat{\gamma}_{l}-\gamma_{0 l}\right)+2 \int_{0}^{1} R_{l}(z)^{2} d z,
\end{aligned}
$$


where $\quad H=\int_{0}^{1} \psi_{l k}^{T}(z) \psi_{l k^{\prime}}^{T}(z)^{T} d z$. Due to $\left\|H_{l}\right\|=o(1)$, after a simple calculation can be

$$
\left(\hat{\gamma}_{l}-\gamma_{0 l}\right)^{T} H\left(\hat{\gamma}_{l}-\gamma_{0 l}\right)=O_{p}\left(n^{\frac{-2 r}{2 r+1}}+a_{n}^{2}\right)
$$

In addition, it is easy to prove $\int_{0}^{1} R_{l}(z)^{2} d z=O_{p}\left(n^{\frac{-2 r}{2 r+1}}\right)$. Therefore $\left\|\hat{g}_{l}(\cdot)-g_{0 l}(\cdot)\right\|^{2}=O_{p}\left(n^{\frac{-r}{2 r+1}}+a_{n}\right), l=1, \cdots, d$.

Proof of Theorem 3.2. According to the SCAD penalty function property and Theorem 3.1 conclusion, it is concluded that when $n \rightarrow \infty$, is sufficient for a given sufficiently small $v=C n^{-r /(2 r+1)}$, and any $\beta_{k}$ satisfying $\quad\left\|\beta_{k}-\beta_{k 0}\right\|=O_{P}\left(n^{\frac{-r}{2 r+1}}\right) \quad$ and $\quad$ any $\quad \gamma_{k} \quad$ satisfying $\left\|\gamma_{k}-\gamma_{k 0}\right\|=O_{p}\left(n^{\frac{-r}{2 r+1}}\right)$, the following two forms are established by probability 1:

$$
\begin{aligned}
& \frac{\partial \eta(\beta, \gamma)}{\partial \beta_{j}}<0, \quad 0<\beta_{j}<v, j=s+1, \cdots, p, \\
& \frac{\partial \eta(\beta, \gamma)}{\partial \beta_{j}}>0, \quad-v<\beta_{j}<0, j=s+1, \cdots, p .
\end{aligned}
$$

According to the above two expressions, the maximum value of $\eta(\beta, \gamma)$ at $\beta_{j}=0, j=s+1, \cdots, p$ just made. By analogy with the proof of Theorem 3.1, we can get

$$
\begin{aligned}
\frac{\partial \eta(\beta, \gamma)}{\partial \beta_{j}} & =\sum_{i=1}^{n} \frac{\partial Q(\beta, \gamma)}{\partial \beta_{j}}+\sum_{i=1}^{n}\left(1-\delta_{i}\right) \frac{\partial m_{Q}\left(Z_{i}, \beta, \gamma\right)}{\partial \beta_{j}}-n p_{\lambda_{1 j}}^{\prime}\left(\left|\beta_{j}\right|\right) \operatorname{sgn}\left(\beta_{j}\right) \\
& \triangleq J_{1}-n p_{\lambda_{1 j}^{\prime}}^{\prime}\left(\left|\beta_{j}\right|\right) \operatorname{sgn}\left(\beta_{j}\right)
\end{aligned}
$$


where

$$
\begin{aligned}
J_{1}= & \sum_{i=1}^{n} \delta_{i} X_{i} \phi_{h}^{\prime}\left[\delta_{i}\left(Y_{i}-X_{i}^{T} \beta-\Psi_{i}^{T} \gamma\right)\right]\left[1+\left(1-\delta_{i}\right) \omega_{n i}(z)\right] \\
= & \sum_{i=1}^{n} \delta_{i} X_{i}\left\{\phi_{h}^{\prime}\left[\delta_{i}\left(\varepsilon_{i}+1_{d}^{T} R\left(Z_{i}\right)\right)\right]+\delta_{i} \phi_{h}^{\prime \prime}\left[\delta_{i}\left(\varepsilon_{i}+1_{d}^{T} R\left(Z_{i}\right)\right)\right]\left[\Psi_{i}^{T}\left(\gamma-\gamma_{0}\right)\right.\right. \\
& \left.+X_{i}^{T}\left(\beta-\beta_{0}\right)\right] \\
& \left.+\frac{1}{2} \delta_{i}^{2} \phi_{h}^{\prime \prime \prime}\left(\delta_{i} \zeta_{i}\right)\left[\Psi_{i}^{T}\left(\gamma-\gamma_{0}\right)+X_{i}^{T}\left(\beta-\beta_{0}\right)\right]^{2}\right\}\left[1+\left(1-\delta_{i}\right) \omega_{n i}(z)\right],
\end{aligned}
$$

where $\zeta_{i}$ is between $\delta_{i}\left[Y_{i}-X_{i}^{T} \beta-\Psi_{i}^{T} \gamma\right]$ and $\delta_{i}\left[\varepsilon_{i}+1_{d}^{T} R\left(Z_{i}\right)\right]$. It is known from Theorem 3.1 that $J_{1}=O_{P}\left(n^{\frac{-r}{2 r+1}}\right)$. Combined with the penalty function, we can get

$$
\frac{\partial \eta(\beta, \gamma)}{\partial \beta_{j}}=-n \lambda_{1 j}\left\{\lambda_{1 j}^{-1} p_{\lambda_{1 j}^{\prime}}^{\prime}\left(\left|\beta_{j}\right|\right) \operatorname{sgn}\left(\beta_{j}\right)+O_{P}\left(\lambda_{1 j}^{-1} n^{\frac{-r}{2 r+1}}\right)\right\}
$$

According to the regular condition (C-7) and $n^{r /(2 r+1)} \lambda_{1 k} \geq n^{r /(2 r+1)} \lambda_{\min }$ $\rightarrow \infty$ the symbol of $\partial \eta(\beta, \gamma) / \partial \beta_{j}$ is determined entirely by the sign of $\beta_{j}$, i.e., formula (8) and (9).

The proof of Theorem 3.2 (ii) still uses a similar proof method.

Proof of Theorem 3.3. From the conclusion of Theorem 3.2, it is found that $n^{r /(2 r+1)}$ consistent local maxima of $\eta\left(\left(\beta_{a}^{T}, 0\right),\left(\gamma_{a}^{T}, 0\right)\right)$ in Theorem 3.3 are at $\hat{\beta}_{a}$ and $\hat{\gamma}_{a}$. According to the process of deriving the maxima, the following two equations are established with probability 1 :

$$
\left.\frac{\partial \eta(\beta, \gamma)}{\partial \beta}\right|_{\beta=\left(\hat{\beta}_{a}^{T}, 0\right)^{T}}=0,\left.\frac{\partial \eta(\beta, \gamma)}{\partial \gamma}\right|_{\gamma=\left(\hat{\gamma}_{a}^{T}, 0\right)^{T}}=0 .
$$

By using the conclusion $\left\|\hat{\beta}-\beta_{0}\right\|=O_{P}\left(n^{\frac{-r}{2 r+1}}+a_{n}\right)=o_{p}(1)$ of Theorem 3.2 , we have $\left|\hat{\beta}_{k}\right|>a \lambda_{1 k}, k=1, \cdots, s$. And by using the conclusion 
$\left\|\hat{\gamma}-\gamma_{0}\right\|=O_{P}\left(n^{\frac{-r}{2 r+1}}+a_{n}\right)=o_{p}(1)$, we have $\left\|\hat{\gamma}_{l}\right\|_{H_{l}}>a \lambda_{2 l}, l=1, \cdots, t$. According to the property of SCAD penalty function, we can get the part of (11) about penalty is 0 , then the upper expression can be expressed as

$$
\begin{aligned}
& \left.\frac{\partial \eta(\beta, \gamma)}{\partial \beta}\right|_{\beta=\left(\hat{\beta}_{a}^{T}, 0\right)^{T}}=\left.\frac{\partial Q(\beta, \gamma)}{\partial \beta}\right|_{\beta=\left(\beta_{a}^{T}, 0\right)^{T}+\left.(1-\delta) \frac{\partial m_{Q}(Z, \beta, \gamma)}{\partial \beta}\right|_{\beta=\left(\beta_{a}^{T}, 0\right)} ^{T}=0,} \\
& \left.\frac{\partial \eta(\beta, \gamma)}{\partial \gamma}\right|_{\gamma=\left(\hat{\gamma}_{a}^{T}, 0\right)^{T}}=\left.\frac{\partial Q(\beta, \gamma)}{\partial \beta}\right|_{\gamma=\left(\gamma_{a}^{T}, 0\right)^{T}+\left.(1-\delta) \frac{\partial m_{Q}(Z, \beta, \gamma)}{\partial \beta}\right|_{\gamma=\left(\gamma_{a}^{T}, 0\right)} ^{T}=0 .}=
\end{aligned}
$$

After calculation, there are

$$
\begin{aligned}
& 0=-\sum_{i=1}^{n} \delta_{i} X_{i a}^{T} \phi_{h}^{\prime}\left[\delta_{i}\left(Y_{i}-X_{i a}^{T} \hat{\beta}_{a}-\Psi_{i a}^{T} \hat{\gamma}_{a}\right)\right]- \sum_{i=1}^{n}\left(1-\delta_{i}\right) \omega_{n i}(z) \delta_{i} X_{i a}^{T} \phi_{h}^{\prime} \\
& \times {\left[\delta_{i}\left(Y_{i}-X_{i a}^{T} \hat{\beta}_{a}-\Psi_{i a}^{T} \hat{\gamma}_{a}\right)\right] } \\
&=-\sum_{i=1}^{n} \delta_{i} X_{i a}\left\{\phi_{h}^{\prime}\left(\delta_{i} \varepsilon_{i}\right)+\delta_{i} \phi_{h}^{\prime \prime}\left(\delta_{i} \varepsilon_{i}\right)\left[1_{d}^{T} R\left(Z_{i}\right)-\Psi_{i}^{T}\left(\hat{\gamma}_{a}-\gamma_{a 0}\right)\right.\right.\left.-X_{i}^{T}\left(\hat{\beta}_{a}-\beta_{a 0}\right)\right] \\
&\left.+\frac{1}{2} \delta_{i}^{T} \phi_{h}^{\prime \prime \prime}\left(\delta_{i} \xi_{i}\right)\left[1_{d}^{T} R\left(Z_{i}\right)-\Psi_{i}^{T}\left(\hat{\gamma}_{a}-\gamma_{a 0}\right)-X_{i}^{T}\left(\hat{\beta}_{a}-\beta_{a 0}\right)\right]^{2}\right\} \\
& \times\left[1+\left(1-\delta_{i} \omega_{n i}(z)\right)\right] \\
& 0 \\
& \begin{array}{c}
\sum_{i=1}^{n} \delta_{i} \Psi_{i a}^{T} \phi_{h}^{\prime}\left[\delta_{i}\left(Y_{i}-X_{i a}^{T} \hat{\beta}_{a}-\Psi_{i a}^{T} \hat{\gamma}_{a}\right)\right]- \\
\sum_{i=1}^{n}\left(1-\delta_{i}\right) \omega_{n i}(z) \delta_{i} \Psi_{i a}^{T} \phi_{h}^{\prime}
\end{array} \\
& \times\left[\delta_{i}\left(Y_{i}-X_{i a}^{T} \hat{\beta}_{a}-\Psi_{i a}^{T} \hat{\gamma}_{a}\right)\right] \\
&=-\sum_{i=1}^{n} \delta_{i} \Psi_{i a}\left\{\phi_{h}^{\prime}\left(\delta_{i} \varepsilon_{i}\right)+\delta_{i} \phi_{h}^{\prime \prime}\left(\delta_{i} \varepsilon_{i}\right)\left[1_{d}^{T} R\left(Z_{i}\right)-\Psi_{i}^{T}\left(\hat{\gamma}_{a}-\gamma_{a 0}\right)\right.\right. \\
&\left.-X_{i}^{T}\left(\hat{\beta}_{a}-\beta_{a 0}\right)\right]
\end{aligned}
$$




$$
\begin{aligned}
+\frac{1}{2} \delta_{i}^{T} \phi_{h}^{\prime \prime \prime}\left(\delta_{i} \xi_{i}\right)\left[1_{d}^{T} R\left(Z_{i}\right)-\Psi_{i}^{T}\left(\hat{\gamma}_{a}-\gamma_{a 0}\right)\right. & \left.\left.-X_{i}^{T}\left(\hat{\beta}_{a}-\beta_{a 0}\right)\right]^{2}\right\} \\
\times & \left.\times 1+\left(1-\delta_{i} \omega_{n i}(z)\right)\right] .
\end{aligned}
$$

Combined with the regular conditions (C-3) and (C-6), we calculate the formula (13)

$$
\hat{\gamma}_{a}-\gamma_{a 0}=\left(\Phi_{n}+o_{p}(1)\right)^{-1}\left\{\Lambda_{n}-\Xi_{n}\left(\hat{\beta}_{a}-\beta_{a 0}\right)\right\}
$$

where $\Lambda_{n}=\frac{1}{n} \sum_{i=1}^{n} \Psi_{i a} \delta_{i}\left[\delta_{i} \phi_{h}^{\prime}\left(\delta_{i} \varepsilon_{i}\right)+\delta_{i} \phi_{h}^{\prime \prime}\left(\delta_{i} \epsilon_{i}\right) 1_{d}^{T} R\left(Z_{i}\right)\right]\left[1+\left(1-\delta_{i}\right) \omega_{n i}(z)\right]$, $\Phi_{n}=\frac{1}{n} \sum_{i=1}^{n} \delta_{i}^{2} \phi_{h}^{\prime \prime}\left(\delta \varepsilon_{i}\right) \Psi_{i a} \Psi_{i a}^{T}\left[1+\left(1-\delta_{i}\right) \omega_{n i}(z)\right], \Xi_{n}=\frac{1}{n} \sum_{i=1}^{n} \delta_{i}^{2} \phi_{h}^{\prime \prime}$ $\left(\delta \varepsilon_{i}\right) \Psi_{i a} X_{i a}^{T}\left[1+\left(1-\delta_{i}\right) \omega_{n i}(z)\right]$.

Combined (12) and (14), we have

$$
\begin{aligned}
& \frac{1}{n} \sum_{i=1}^{n} \delta_{i}^{2} X_{i a} \phi_{h}^{\prime \prime}\left(\delta_{i} \varepsilon_{i}\right)\left[X_{i a}-\Xi_{n}^{T}\left(\Phi_{n}^{-1}+o_{p}(1)\right) \Psi_{i}\right]^{T}\left(\hat{\beta}_{a}-\beta_{a 0}\right) \\
& =\frac{1}{n} \sum_{i=1}^{n} \delta_{i} X_{i a}\left[\phi_{h}^{\prime}\left(\delta_{i} \varepsilon_{i}\right)+\delta_{i} \phi_{h}^{\prime \prime}\left(\delta_{i} \varepsilon_{i}\right) 1_{d}^{T} R\left(Z_{i}\right)-\delta_{i} \phi_{h}^{\prime \prime}\left(\delta_{i} \varepsilon_{i}\right) \Psi_{i a}^{T}\left(\Phi^{-1}+o_{p}(1)\right) \Lambda_{n}\right] .
\end{aligned}
$$

Note the following facts

$$
\begin{aligned}
& E\left(\frac{1}{n} \sum_{i=1}^{n} \phi_{h}^{\prime \prime}\left(\delta_{i} \varepsilon_{i}\right) \Xi_{n}^{T} \Phi_{n}^{-1} \Psi_{i a}\left[X_{i a}^{T}-\Psi_{i a}^{T} \Phi_{n}^{-1} \Xi_{n}^{T}\right]\right)=0, \\
& \operatorname{Var}\left(\frac{1}{n} \sum_{i=1}^{n} \phi_{h}^{\prime \prime}\left(\delta_{i} \varepsilon_{i}\right) \Xi_{n}^{T} \Phi_{n}^{-1} \Psi_{i a}\left[X_{i a}^{T}-\Psi_{i a}^{T} \Phi_{n}^{-1} \Xi_{n}^{T}\right]\right)=o_{p}(1) .
\end{aligned}
$$


Therefore, in conjunction with (15), we have

$$
\begin{aligned}
&\left\{\frac{1}{n} \sum_{i=1}^{n} \delta_{i}^{2} \phi_{h}^{\prime \prime}\left(\delta_{i} \varepsilon_{i}\right) \check{X}_{i a} \check{X}_{i a}^{T}+o_{p}(1)\right\} \sqrt{n}\left(\hat{\beta}_{a}-\beta_{a 0}\right) \\
&=\frac{1}{\sqrt{n}} \sum_{i=1}^{n} \delta_{i} \check{X}_{i a} \phi_{h}^{\prime}\left(\delta_{i} \varepsilon_{i}\right)+\frac{1}{\sqrt{n}} \sum_{i=1}^{n} \delta_{i}^{2} \check{X}_{i a} \phi_{h}^{\prime \prime}\left(\delta_{i} \varepsilon_{i}\right) 1_{d}^{T} R\left(Z_{i}\right) \\
&\left.\quad-\frac{1}{\sqrt{n}} \sum_{i=1}^{n} \delta_{i}^{2} \check{X}_{i a} \phi_{h}^{\prime \prime}\left(\delta_{i} \epsilon_{i}\right) \Psi_{i a}^{T}\left(\Phi^{-1}+o_{p}(1)\right) \Lambda_{n}\right] \\
& \triangleq G_{1}+G_{2}+G_{3},
\end{aligned}
$$

where $\check{X}_{i a}=X_{i a}-\Xi_{n}^{T} \Phi_{n}^{-1} \Psi_{i a}$. And because of

$$
\sum_{i=n}^{n} \delta_{i}^{2} \check{X}_{i} \phi_{h}^{\prime \prime} \Psi_{i a}^{T}=\sum_{i=n}^{n} \delta_{i}^{2} X_{i} \phi_{h}^{\prime \prime} \Psi_{i a}^{T}-\Xi_{n}^{T} \Phi_{n}^{-1} \sum_{i=1}^{n} \delta_{i}^{2} \Psi_{i} \Psi_{i}^{T}=0
$$

so there is $G_{3}=0$. Also because of $\left\|R\left(Z_{i}\right)\right\|=O_{p}\left(n^{\frac{-r}{2 r+1}}\right)=o_{p}(1)$, we has $G_{2}=o_{p}(1)$. Then, the (16) formula can be expressed as

$$
\left\{\frac{1}{n} \sum_{i=1}^{n} \delta_{i}^{2} \phi_{h}^{\prime \prime}\left(\delta_{i} \varepsilon_{i}\right) \check{X}_{i a} \check{X}_{i a}^{T}+o_{p}(1)\right\} \sqrt{n}\left(\hat{\beta}_{a}-\beta_{a 0}\right)=\frac{1}{\sqrt{n}} \sum_{i=1}^{n} \delta_{i} \check{X}_{i a} \phi_{h}^{\prime}\left(\delta_{i} \varepsilon\right)+o_{p}(1) .
$$

Next, we can use the large number theorem and the central limit theorem.

$$
\begin{aligned}
& \frac{1}{n} \sum_{i=1}^{n} \delta_{i}^{2} \phi_{h}^{\prime \prime}\left(\delta_{i} \varepsilon_{i}\right) \check{X}_{i a} \check{X}_{i a}^{T} \stackrel{p}{\rightarrow} \sum_{a}, \\
& \sqrt{n} \sum_{i=1}^{n} \delta_{i} \phi_{h}^{\prime}\left(\delta_{i} \varepsilon_{i}\right) \check{X}_{i a} \stackrel{d}{\rightarrow} N\left(0, \Omega_{a}\right) .
\end{aligned}
$$

Combined with (17)-(19) formula and Slutsky's theorem, it is easy to get

$$
\sqrt{n}\left(\hat{\beta}_{a}-\beta_{a 0}\right) \stackrel{d}{\rightarrow} N\left(0, \sum_{a}^{-1} \Omega_{a} \sum_{a}^{-1}\right) .
$$


The following proof (19) holds. Any vector $\zeta$ that is not completely zero,

$$
\zeta^{T} G_{1}=\sum_{i=1}^{n} \frac{1}{\sqrt{n}} \zeta^{T} \phi_{h}^{\prime}\left(\delta_{i} \varepsilon\right) \check{X}_{i a} \triangleq \sum_{i=1}^{n} a_{i} \xi_{i},
$$

where $a_{i}^{2}=\frac{1}{n} G\left(X_{i}, Z_{i}, h\right) \zeta^{T} \check{X}_{i a} \check{X}_{i a}^{T} \zeta, \xi_{i}$ are independent random variable, and under given $C$ condition, its mean value is 0 and the variance is 1 . The following verifies the Lindeberg central limit theorem condition. If

$$
\frac{\max _{i} a_{i}^{2}}{\sum_{i=1}^{n} a_{i}^{2}} \stackrel{p}{\rightarrow} 0
$$

there is $\sum_{i=1}^{n} a_{i} \xi_{i} / \sqrt{\sum_{i=1}^{n} a_{i}^{2}} \stackrel{d}{\rightarrow} N(0,1)$, thus (19) form. The following certificate (20) is established.

Because of $\left(\zeta^{T} \check{X}_{i a}^{T}\right)^{2} \leq\|\zeta\|^{2}\left\|\check{X}_{i a}\right\|^{2}$, so $\quad a_{i}^{2} \leq n^{-1} G\left(X_{i}, Z_{i}, h\right)\|\zeta\|^{2}$ $\left\|\check{X}_{i a}\right\|^{2}$. Combined with $\left\|\check{X}_{i a}\right\|=\left\|X_{i a}-\Xi^{T} \Phi^{T} \Psi_{i a}\right\| \leq\left\|X_{i a}\right\|+\left\|\Xi^{T} \Phi^{T} \Psi_{i a}\right\|$, and regular conditions (C-3), we can get $\max _{i}\left\|X_{i}\right\| / \sqrt{n}=o_{p}(1)$, so there are $\max _{i}\left\|\check{X}_{i} / \sqrt{n}=o_{p}(1)\right\|$. Application of Slutsky theorem again, then (20) holds. Thus, Theorem 3.3 proves complete.

\section{References}

[1] H. Lian, Variable selection in high-dimensional partly linear addition models, Journal of Nonparametric Statistics 24(4) (2012), 825-839.

DOI: https://doi.org/10.1080/10485252.2012.701300

[2] H. Noh, K. Chung and Ingrid Van Keilegom, Variable selection of varying coefficient models in quantile regression, Electronic Journal of Statistics 6 (2012), 1220-1238.

DOI: https://doi.org/10.1214/12-EJS709

[3] J. Guo, M. Tang, M. Tian and K. Zhu, Variable selection in high-dimensional partially linear additive models for composite quantile regression, Computational Statistics and Data Analysis 65 (2013), 56-67.

DOI: https://doi.org/10.1016/j.csda.2013.03.017 
[4] R. Zhang, W. Zhao and J. Liu, Robust estimation and variable selection for semiparametric partially linear varying coefficient model based on modal regression, Journal of Nonparametric Statistics 25(2) (2013), 523-544.

DOI: https://doi.org/10.1080/10485252.2013.772179

[5] H. Yang, J. Lv and C. Guo, Robust estimation and variable selection for varyingcoefficient single-index models based on modal regression, Communications in Statistics - Theory and Methods 45(14) (2016), 4048-4067.

DOI: https://doi.org/10.1080/03610926.2014.915043

[6] J. Lv, H. Yang and C. Guo, Variable selection in partially linear additive models for modal regression, Communications in Statistics-Simulation and Computation 46(7) (2017), 5646-5665.

DOI: http://dx.doi.org/10.1080/03610918.2016.1171346

[7] P. X. Zhao and L. G. Xue, Variable selection for semiparametric varying-coefficient partially linear models with missing response at random, Acta Mathematica Sinica, English Series 27(11) (2011), 2205-2216.

DOI: https://doi.org/10.1007/s10114-011-9200-1

[8] H. Yang and X. Xia, Variable selection for semiparametric varying coefficient partially linear errors-in-variables (EV) model with missing response, Communications in Statistics - Theory and Methods 44(21) (2015), 4521-4539.

DOI: https://doi.org/10.1080/03610926.2013.791376

[9] X. Ding, J. Chen and Xiaoqin Zhu, Parameter estimation of quantile regression with responses random missing, Statistics and Decision 6 (2018), 65-67.

DOI: https://doi.org/10.13546/j.cnki.tjyjc.2018.06.015

[10] W. Yao, B. G. Lindsay and Runze Li, Local modal regression, Journal of Nonparametric Statistics 24(3) (2012), 647-663.

DOI: https://doi.org/10.1080/10485252.2012.678848

[11] J. Fan and R. Li, Variable selection via nonconcave penalized likelihood and its oracle properties, Journal of the American Statistical Association 96(456) (2001), 1348-1360.

DOI: https://doi.org/10.1198/016214501753382273

[12] W. Zhao, R. Zhang, J. Liu and Y. Lv, Robust and efficient variable selection for semiparametric partially linear varying coefficient model based on modal regression, Annals of the Institute of Statistical Mathematics 66(1) (2014), 165-191.

DOI: https://doi.org/10.1007/s10463-013-0410-4

[13] C. de Boor, A Practical Guide to Splines, Springer, New York, 2001. 